\title{
Aplicaciones y ejemplos de modelos de crecimiento diamétrico para árboles tropicales
}

\section{Applications and examples of diameter growth models for tropical trees}

\author{
Pedro A. Domínguez-Calleros ${ }^{1}$, Felipa de J. Rodríguez-Flores ${ }^{2}$, Liliana Lizárraga-Mendiola ${ }^{3}$, Marco A. \\ Jiménez-Gómez ${ }^{4}$, Jose Navar ${ }^{4 *}$ \\ ${ }^{1}$ Facultad de Ciencias Forestales. Universidad Juárez del Estado de Durango Av. Río Papalopan s/n. Colonia. Valle del Sur. \\ CP. 34600. Durango, Mexico \\ ${ }^{2}$ Universidad Politecnica de Durango. Carretera Durango-Mexico Km 9.5. Localidad Dolores Hidalgo. CP. 34300. Durango, \\ Mexico \\ ${ }^{3}$ Universidad Autónoma del Estado de Hidalgo. Area Académica de Ingeniería. Mineral de la Reforma, Pachuca, Hidalgo, \\ México \\ ${ }^{4}$ Tecnológico Nacional de México. Instituto Tecnológico de Ciudad Victoria. Blvd Emilio Portes Gil No 1301 Pte. Cd Victoria, \\ CP. 87010, Tamaulipas, México \\ *Autor de correspondencia: jose.navar@itvictoria.edu.mx
}

Artículo científico recibido: 07 de abril de 2016, aceptado: 21 de octubre de 2016

RESUMEN. Los modelos de incremento y rendimiento forman la base de la planeación estratégica forestal convencional y sustentable. Los objetivos fueron ajustar cinco modelos de incremento en diámetro a árboles individuales creciendo en bosques tropicales Latinoamericanos y desarrollar las curvas de crecimiento en función de la edad a partir de los modelos de incremento. Se usaron incrementos diamétricos anuales de árboles individuales de especies tropicales de Latinoamérica. Con técnicas estadísticas se ajustaron los modelos de incremento: Log Normal, Chapman-Richards, Von Bertalanfy, Logístico y Gompertz con ecuaciones integrables en tiempo. Las curvas de crecimiento en diámetro se desarrollaron suponiendo el diámetro inicial a una edad de un año. Los resultados muestran los parámetros de las ecuaciones de mejor ajuste y las curvas de crecimiento diamétrico en función de la edad y se describe su potencial utilización en el desarrollo de planes de manejo forestal. La base de datos no fue suficiente para detectar si el número de árboles muestreados influyó en el ajuste de un modelo en específico. Es necesario realizar nuevas investigaciones para revisar los límites donde aplican las suposiciones con el ajuste de los modelos a series de tiempo de crecimiento convencionales y a series aún más cortas en tiempo.

Palabras clave: Chapman-Richards, log normal, Von Bertalanffy, Gompertz, logistic

ABSTRACT. Growth and yield models of individual tropical trees are central to conventional and sustainable forest management. The objective was to fit five diameter growth and yield models to Latin-American individual tropical trees and to derive diameter growth curves as a function of age. Diameter increments were collected from individual tropical trees. With the use of statistical techniques, five increment models amenable to be integrated over time: Log Normal, Chapman-Richards, Von Bertalanfy, Logistic, and Gompertz were fitted. Assuming an initial diameter of seedlings at age $=1$, diameter growth curves were developed, which can also be fitted by the integral form of the increment equations. Results show parameters of best adjusted increment equations as well as growth curves as a function of age and the document discusses their potential utilization in forest management plans. The goodness of fit statistics could not tell apart whether the number of monitored trees controlled the best fitting model. Further research is recommended to check on the limits of model application and the assumptions set on the diameter growth time series.

Key words: Chapman-Richards, log normal, Von Bertalanffy, Gompertz, logistic 
Dominguez-Calleros et al.

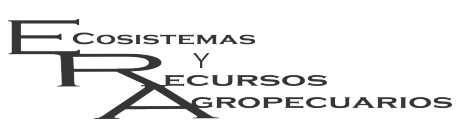

Crecimiento diamétrico de árboles tropicales

Ecosist. Recur. Agropec. 4(11):265-274,2017

\section{INTRODUCCIÓN}

Los cálculos del incremento y rendimiento de los árboles individuales son centrales en el manejo de ecosistemas forestales. Estos son necesarios para calcular el volumen a remover, el tiempo entre intervenciones, las características de la masa forestal futura, la productividad de los ecosistemas y de los almacenes, y el flujo de varios elementos biogeoquímicos (Clutter et al. 1983). El crecimiento de un árbol o una masa forestal es el cambio irreversible en las dimensiones durante periodos definidos de tiempo (Vanclay 1995). Este proceso es dinámico en un bosque, comprende un balance de masas que incluye una entrada, una tasa de cambio o movimiento y una salida. Suponiendo que la masa forestal se encuentra bajo manejo, la incorporación se cuantifica con el número de individuos que se anexan a la masa forestal al alcanzar las dimensiones inventariable (Clutter et al. 1983).

La mortalidad de árboles ocurre en forma natural por procesos determinísticos explicados por la competencia y la edad, por procesos estocásticos explicados por perturbaciones aleatorias como los incendios, plagas, enfermedades, intervenciones forestales, entre otros (Alder y Synnott 1992). El crecimiento de los árboles es el cambio gradual del diámetro, altura, área basal y como consecuencia el volumen y la masa; es afectado por diversos componentes, entre los que destacan el origen de la especie y su entorno ambiental (Smith et al. 1992). El crecimiento de los árboles y los bosques muestran el componente determinístico, el cual es modelado por la clásica curva sigmoidal (Zeide 1999) y el componente estocástico o aleatorio dado por las variaciones ambientales cambiantes entre años o periodos de tiempo (Návar y Lizárraga-Mendiola 2014).

El crecimiento de árboles individuales cuando se monitorea por periodos cortos de tiempo, se denomina incremento, el cual considera los cambios en las dimensiones; pero no la competencia, reclutamiento ni mortalidad (Peng 2000). Se sabe que el crecimiento empírico de árboles individuales, es difícil de estimar y proyectar, por qué depende de procesos fisiológicos, climáticos, físicos y biológicos (Van- clay 1995). Sin embargo, el crecimiento muestra un comportamiento definido, el cual puede ser modelado de forma matemática y evaluado con modelos no lineales (Zeide 1999). Las oscilaciones aleatorias por otro lado se pueden proyectar de forma empírica con modelos estocásticos y por medio de técnicas de asociación (Haan 2003). El comportamiento sigmoidal clásico forma la base del modelaje convencional del crecimiento de árboles individuales y de masas forestales. El componente aleatorio debe de ser parte integral de los estudios de crecimiento, ya que no se evalua en los modelos de crecimiento (Clutter et al. 1983).

Existen varias técnicas para modelar la curva sigmoidal del crecimiento de árboles y de masas forestales. Los modelos se pueden clasificar en modelos a nivel del rodal, de clases de árboles y de árboles individuales (Peng 2000, Husch et al. 2003, Buongiorno 2004). Los tres modelos son aplicables a bosques regulares 0 irregulares, los primeros han sido desarrollados en bosques de México (NávarCháidez y González-Elizondo 2009, Návar-Cháidez y Domínguez-Calleros 2013, Návar et al. 2016). El crecimiento de los árboles individuales se ha modelado con restricciones en la dependencia o independencia de la distancia con los árboles vecinos, como los de Clutter et al. (1983) y Vanclay (1995) para bosques de otros países y por Corral-Rivas y NávarCháidez (2005) para bosques templados de México.

Los datos temporales de incrementos medidos en árboles individuales son escasos, por el corto tiempo que tienen las numerosas parcelas de monitoreo permanente que se establecen; aunque cada vez se tienen más publicaciones con datos de incrementos del diámetro de árboles tropicales (López-Ayala et al. 2006, Ramírez-Angulo et al. 2009, Lozano et al. 2012). Diversos modelos cumplen con los requisitos al proyectar el incremento y crecimiento, como el Log Normal, ChapmanRichards, Von Bertalanffy, Logístico, Gompertz, Hossfeld, Monomolecular, Korf, Weibull, Sloboda, Levakovick, Schumacher, entre otros (Zeide 1995).

Los modelos de árboles individuales orientados al manejo, son modelos empíricos por naturaleza (Peng 2000). Estos son independientes de la dis- 


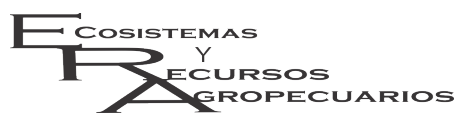

Dominguez-Calleros et al.

Crecimiento diamétrico de árboles tropicales

Ecosist. Recur. Agropec. 4(11):265-274,2017

tancia, cuando los datos se componen de un simple listado de árboles con sus dimensiones y sus incrementos o crecimientos (Husch et al. 2003). Dependen de la distancia cuando se conoce la posición geográfica de los árboles y se estima un índice de competencia de copa para cada árbol, para determinar si el árbol muere o sobrevive a la competencia. Si el árbol sobrevive se estima su incremento en diámetro, altura, área basal, y dimensión de copa, en función de la competencia (Vanclay 1995). El incremento y crecimiento de los árboles tropicales es más complejo, porque no cuentan con anillos de crecimiento visibles con técnicas convencionales. Por esta razón, las mediciones de los incrementos anuales son la base del desarrollo de los modelos de crecimiento. Desafortunadamente muchas de las investigaciones adolecen del ajuste de modelos de incremento y en mucho menor medida en el desarrollo de las clásicas curvas de crecimiento. El desarrollo, aplicación y validación de los modelos de incremento y rendimiento requieren de datos en su construcción. Al respecto Alder y Synnott (1992) y Vanclay (1995) señalan que existen varias posibilidades de obtener datos cuantitativos, dentro de los cuales destacan los sitios permanentes de monitoreo; la remedición de sitios temporales; el análisis troncal en conjunto con datos de inventarios; las crono-secuencias de rodales o árboles y las mediciones por periodos cortos de tiempo en rodales o árboles individuales de diferente edad. Actualmente existen parcelas permanentes de monitoreo forestal para entender la dinámica de los árboles y las masas forestales (FAO 2015). Para México se han reportado más de 3000 parcelas establecidas entre el 2004 y 2006, las cuales fueron medidas y se encuentran en proceso de una segunda remedición (INF 2012).

Se han reportado técnicas para modelar el incremento y crecimiento (Návar-Cháidez y Domínguez-Calleros 2013). Al respecto Návar (2014) presentó un modelo para árboles de bosques irregulares con el uso de la distribución Weibull, donde define de forma los ritmos de crecimiento de los pinos y los encinos. Sin embargo, son escasos los modelos de incremento y rendimiento para especies a las que no se les pueden cuantificar los anillos de crecimiento con las técnicas convencionales, como la mayoría de las especies tropicales. Por lo anterior, el objetivo del presente estudio fuer colectar de la literatura científica los estudios y mediciones de incremento en diámetro de especies tropicales de Latinoamérica y ajustar cinco modelos no lineales, para desarrollar curvas de crecimiento en diámetro.

\section{MATERIALES Y MÉTODOS}

Se colectaron datos de los incrementos diamétricos de árboles tropicales de Latinoamérica; para luego tamizar la información y seleccionar aquella que tuviera suficientes árboles monitoreados y mostrara una relación clara del incremento diamétrico (ID) anual y el diámetro normal (D) del árbol. Para luego ajustar los datos a los modelos del incremento diamétrico en función del diámetro normal del árbol; para elaborar las curvas de crecimiento en diámetro, con la suposición de dos diámetros iniciales a la edad de un año.

Se realizó una buqueda en google con las palabras incremento, diámetro, árboles tropicales, América Latina. El tamizado permitió seleccionar aquellos que tuvieran una base de datos de incremento diamétrico de al menos cinco árboles distribuidos en un rango de dimensiones en diámetro normal coherente con la típica curva del incremento diamétrico. Para el ajuste de los modelos de incremento diamétrico y las curvas de crecimiento se consultaron las publicaciones de Zeide (1995), Ricker y del Río (2004) y Merlín-Bermúdez y NávarCháidez (2005), quienes ajustaron la derivada de los típicos modelos de crecimiento a los incrementos diamétricos de especies difíciles de cuantificar los anillos de crecimiento. Se probaron cinco modelos de ID en función de D (Tabla 1). El cálculo de las curvas de crecimiento se realizó suponiendo un diámetro inicial a la edad, $\mathrm{t}=1$ año de $1 \mathrm{~cm}$ y de $10 \mathrm{~cm}$ para conocer la sensibilidad de la curva a estos dos valores supuestos. Una vez establecido Do a un año, se proyectó el incremento diamétrico con la ecuación de incremento para obtener la curva respectiva.

Las ecuaciones tienen las propiedades que en 
Tabla 1. Modelos de incremento y crecimiento para árboles y masas forestales.

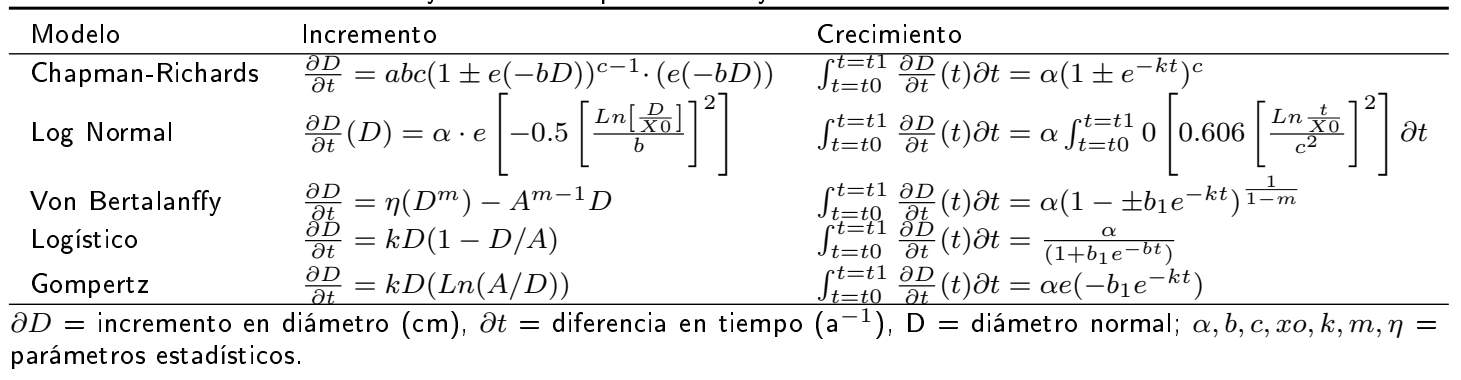

su forma derivada predicen el incremento instantáneo en función de las dimensiones del arbolado, en su forma integral en tiempo resultan en la clásica curva del crecimiento en diámetro en función de la edad de los árboles o masas forestales y la mayoría de los modelos parten de un incremento corriente anual de cero cuando el diámetro es $=0$. El diámetro normal esta relacionado con la edad y considerando un incremento inicial, por un año, con un diámetro inicial Do, el ajuste de las ecuaciones de incremento resulta en el modelo de crecimiento en función de la edad y no de las dimensiones del arbolado. Este procedimiento ha sido desarrollado y explicado por Ricker y Del Rio (2004) para especies de selvas tropicales y por Merlín-Bermúdez y NávarCháidez (2005) para encinos de bosques templados del norte de México.

Los cinco modelos (Tabla 1) se ajustaron a los ID en función de D para la especie Anacardium excelsum de bosques tropicales Colombianos (Lozano et al. 2012) para ver el ajuste de los modelos de ID y como se comparan una vez ajustados y aplicados los modelos entre ellos. Posteriormente se ajustaron los modelos a los datos de incrementos diamétricos de Liquidambar stryaciflua, Quercus germana y Quercus xalapensis de bosques nublados (Williams-Linera 1996); Bursera simaruba, Tabebuia donnell-smithii y Cordia elaeagnoides de bosques estacionales (López-Ayala et al. 2006); y para 25 especies tropicales medidas en los bosques estacionales de Venezuela (Ramírez-Angulo et al. 2009). Solamente se reportan los modelos de mejor ajuste para todas estas especies con excepción del primer caso. También se probó el modelo no lineal de Chapman-Richards en su forma integrada a datos diamétricos provenientes de análisis troncales, como una forma de explicar cómo se modelan los ritmos de crecimiento para un conjunto de árboles de forma simultánea con diámetro conocido.

\section{RESULTADOS}

Modelos de incremento y derivación de los modelos de crecimiento de Anacardum excelsum

Los incrementos diamétricos de Anacardium excelsum muestran la diversidad de incrementos existentes en los árboles muestreados (Figura 1). Se observan diferencias entre los modelos, sobre todo en las etapas primarias y tardías del crecimiento. Los modelos Log-Normal, Gompertz y Von Bertalanffy predicen mayores tasas de incremento iniciales con menores tasas de incremento final. Como consecuencia los modelos proyectan crecimientos diamétricos altos al inicio, pero convergen en la clásica asíntota del diámetro en tiempo. A pesar de la buena consistencia en las proyecciones del diámetro para estas ecuaciones, el modelo que presentó el mejor ajuste fue el de Chapman-Richards, con los siguientes parámetros:

$\frac{\partial D}{\partial t}=2125 \cdot 0.0166 \cdot 0.2 \cdot(1-\exp (-0.0166 D))^{0.8}$
$(\exp (-0.0166 D)), r^{2}=0.68, n=12$.

Las características de las ecuaciones hacen que las proyecciones tengan que ser verificadas con datos diamétricos de árboles de la especies de parcelas permanentes de monitoreo o de parcelas aban- 

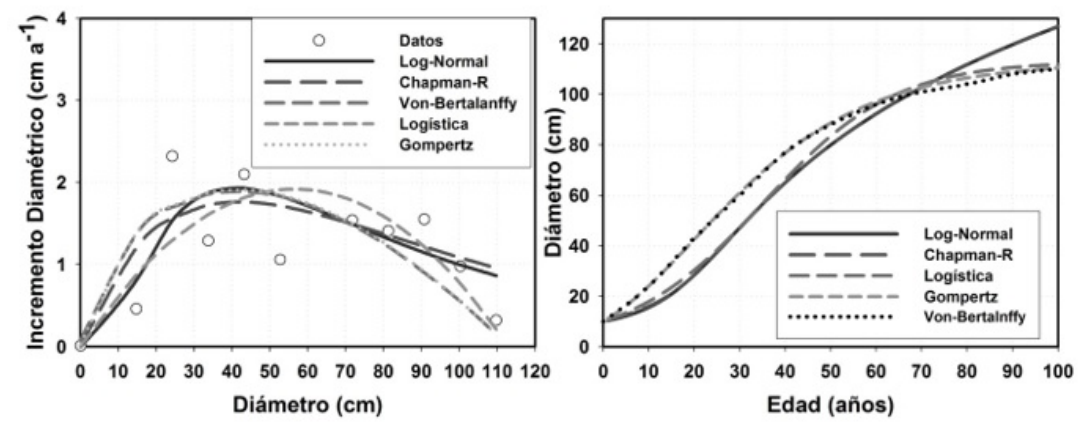

Figura 1. Ajuste y proyección de los modelos Log-Normal, Chapman-Richards, Von Bertalanffy, Logístico y Gompertz al incremento diamétrico de Anacardium excelsum (Kunth) Skeels.

donadas, donde se conoce con exactitud la edad de los árboles o el tiempo desde la regeneración o establecimiento y abandono de los sitios. Un promedio de todos estos modelos provee una mejor estimación de cada una de las curvas individuales como ha sido probado con la teoría del límite central. Las curvas proyectan un incremento promedio y la desviación de este promedio a cada uno de los datos se consideran oscilaciones aleatorias que se pueden modelar por el genotipo y el medio ambiente.

\section{Bosques nublados}

La diversidad de crecimientos y como consecuencia de los incrementos corrientes anuales en diámetro para varias especies del bosque nublado, se observan en la Figura 2. Las especies que destacan por el número de sus mediciones en varios rangos diamétricos son Liquidambar stryaciflua, Quercus germana y Quercus xalapensis. Los resultados de la simulación del crecimiento diamétrico utilizando los modelos que proyectaron los mejores estadísticos, para cada una de las tres especies se presentan a continuación:

Quercus germana: $\frac{\partial D}{t}(D)=2.3 \exp \left[-0.5\left[\frac{\ln \frac{D}{3.0}}{0.458}\right]^{2}\right]$, $r^{2}=0.50, n=10$

Liquidambar stryaciflua: $\frac{\partial D}{\partial t}=390 \cdot 0.0292 \cdot 0.8$. $(1-\exp (-0.0292 D))^{0.2} \cdot(\exp (-0.0292 D)), r^{2}=$ $0.71, n=9$
Quercus xalepensis: $\frac{\partial D}{\partial t}=898 \cdot 0.021 \cdot 0.1$. $(1-\exp (-0.021 D))^{0.9} \cdot(\exp (-0.021 D)), r^{2}=$ $0.30, n=8$

Los modelos de la Figura 2 muestran que el género Quercus alcanza entre 20 y $50 \mathrm{~cm}$ de diámetro normal a los 100 años de edad. Mientras que el género Liquidambar, alcanza los $100 \mathrm{~cm}$ de diámetro a los 80 años.

\section{Bosques secos}

Los incrementos diámetricos de Bursera simaruba, Tabebuia donnell-smithii y Cordia elaeagnoides de la selva mediana sub-caducifolia son variables (Figura 3). Con la información recabada con las bandas de crecimiento y forzando la curva a salir del origen (0), y suponiendo que los centros de las clases diamétricas son 10, 15, 26.3 y 45 $\mathrm{cm}$ se evaluaron los incrementos diamétricos con el modelo de Chapman-Richards, el cual presento los siguientes parámetros:

Bursera simaruba $\frac{\partial D}{\partial t}=376.8 \cdot 0.0167 \cdot 0.20 \cdot(1-$ $\left.\exp (-0.0167 D))^{0.8} \cdot \exp (-0.0167 D)\right)$

Tabebuia donell-smithii $\frac{\partial D}{\partial t}=632.0 .0257 \cdot 0.2$. $(1-\exp (-0.0257 D))^{0.8} \cdot(\exp (-0.0257 D)), n=$ $5, r^{2}=0.45$

Cordia eleagnoides $\frac{\partial D}{\partial t}=87.62 \cdot 0.049 \cdot 0.2 \cdot(1-$ 


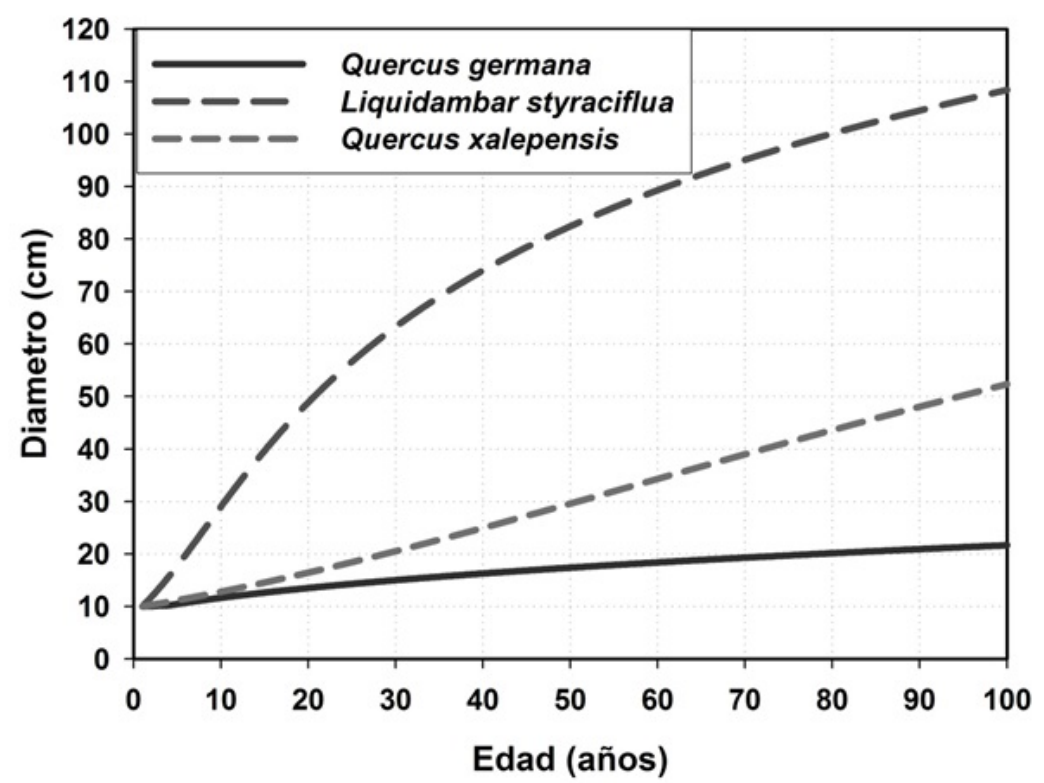

Figura 2. Crecimientos diamétricos simulados con curvas empíricas ajustadas a los incrementos diamétricos para tres especies.

$\exp (-0.049 D))^{0.8} \cdot(\exp (-0.049 D)) n=5, r^{2}=$ 0.78

Se presentan los datos y modelos de incremento diamétrico en la Figura 3. Los crecimientos diamétricos proyectados con el modelo de ChapmanRichards suponiendo un diámetro inicial Do de 10 $\mathrm{cm}$ se presentan en la Figura 4. Los géneros Bursera y Cordia alcanzan cerca de $30 \mathrm{~cm}$ de diámetro a los 100 años de edad. Por otra parte, el género Tabebuia alcanza los $80 \mathrm{~cm}$ de diámetro a los 100 años.

\section{Bosques Estacionales}

Se utilizaron datos sobre incrementos diamétricos instantáneos de 25 especies que crecen en los bosques estacionales de los Llanos Occidentales de Venezuela. De los cinco modelos ajustados el que mejor se ajusto fue el modelo Log Normal, con los parámetros:

$\frac{\partial D}{\partial t}(D)=0.3923 \exp \left[-0.5\left[\frac{\ln \left[\frac{D}{22.77}\right]}{0.7724}\right]^{2}\right] ; r^{2}=$
$0.94, n=25$
El modelo se integró para desarrollar el

www.ujat.mx/era crecimiento en diámetro, con Do $=10 \mathrm{~cm}$, los resultados se presentan en la Figura 5a. En general, se alcanzan los $45 \mathrm{~cm}$ de diámetro normal a los 100 (Figura 5b).

\section{Datos de análisis troncales}

El crecimiento de un árbol individual es relativamente fácil de modelar de forma analítica o empírica. Se presenta un modelo de crecimiento para 29 árboles individuales. El modelo calcula el diámetro sin corteza (Dsc) y utiliza el diámetro a 20 años $\left(D_{20}\right)$ de edad como covariable de anclaje del modelo integrado de Chapman-Richards de tres parámetros. La bondad de ajuste del modelo es adecuada de acuerdo con la ecuación:

$D_{S C}=D_{20} \cdot 7.76(1-\exp (-0.0062 t))^{0.9935}, r^{2}=$ $0.97, S x=2.2 \mathrm{~cm}$

\section{DISCUSIÓN}

Los modelos de incremento y crecimiento deben de tomarse con precaución, ya que dan 

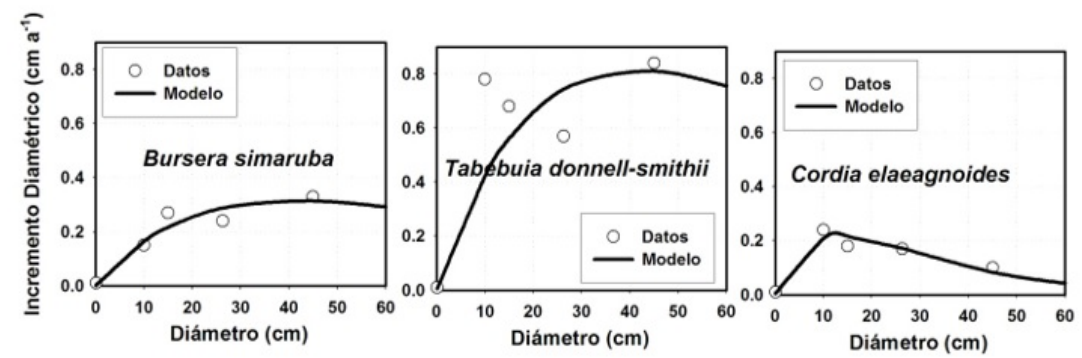

Figura 3. Incrementos diamétricos de tres especies de la selva mediana subcaducifolia.

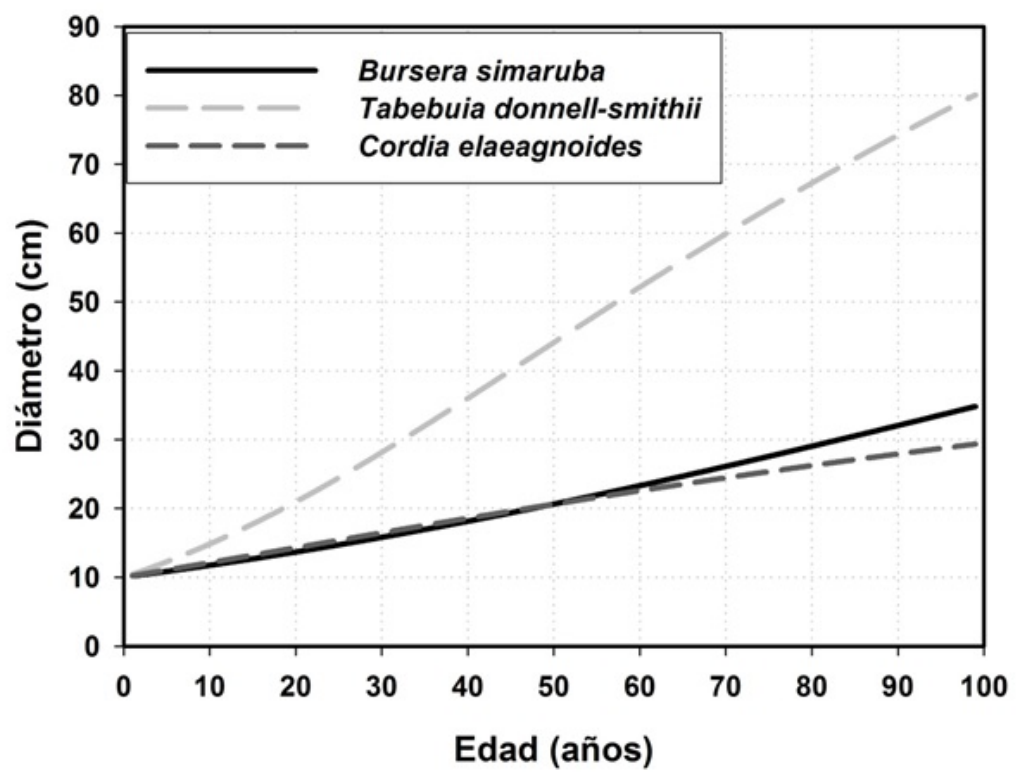

Figura 4. Crecimiento en diámetro derivado del modelo de Chapman-Richards para los incrementos diamétricos de tres especies de la selva mediana subcaducifolia.

crecimientos diamétricos a la baja para algunas especies, sobre todo en etapas tempranas del crecimiento. Los incrementos diamétricos surgen del $\mathrm{Do}=0$ o de $\mathrm{Do}=10 \mathrm{~cm}$, el procedimiento no es sensible a los diámetros calculados a partir de los 50 años de edad, porque se acelera de forma rápida hasta alcanzar un máximo en etapas secundarias del crecimiento. La falta de datos y modelos que predicen de forma adecuada la tendencia inicial del ID sesgan a la baja los crecimientos en los primeros 50 años; otras posibles causas de la subestimación puede ser el sesgo de los centros de clase.
Para mejorar el ajuste de los modelos y predecir de forma más precisa los ID y D se sugiere que, aunque el diámetro inicial Do de las plántulas no parece afectar el $D$ después de los 50 años, se seleccione de forma cuidadosa; tener una mayor fuente de datos con clases diamétricas mayores, para entender los incrementos en las etapas tardías, ya que es posible que no disminuyan como lo indican los modelos. En las clases diamétricas menores se recomienda medir el diámetro basal y correlacionarlo con el diámetro normal para modelar de forma adecuada la aceleración inicial de la ecuación de crecimiento. 

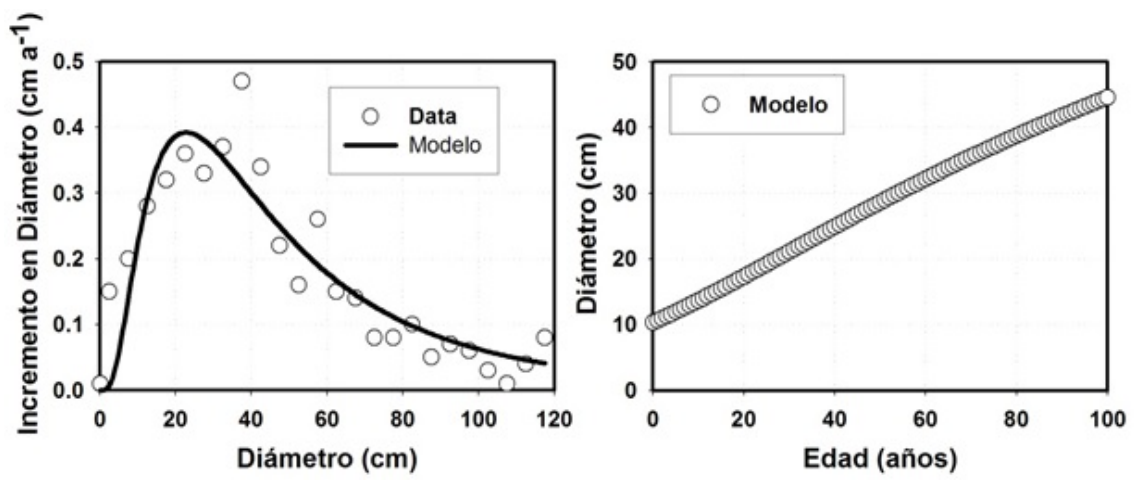

Figura 5. Incremento y rendimiento en diámetro para varias especies de bosques estacionales.

Además de entender que la curva proyecta el incremento promedio instantáneo, y que las variaciones pueden ser consecuencia del medio ambiente, por lo que deben ser tratadas como variables aleatorias (Ricker y del Rio 2004).

A pesar de estas variaciones en el ajuste de los modelos, se presentan los crecimientos diamétricos de los árboles estudiados y sus diferencias en proyecciones de 100 años. Los árboles tropicales parecen alcanzar en general mayores dimensiones en el diámetro normal que los encinos provenientes de bosques templados (Návar-Cháidez y DomínguezCalleros 2013, Návar 2014). Estas diferencias se encuentran asociadas a la mayor productividad de los sitios tropicales que los de bosques templados (Merlín-Bermúdez y Návar-Cháidez 2005). Dentro de los mismos bosques tropicales existen diferencias en los ritmos de crecimiento de las especies que componen las masas forestales. Por ejemplo, en las selvas medianas, Tabebuia donell-smithii crece a un ritmo dos veces mayor que las especies Cordia elaeagnoides y Bursera simaruba (López-Ayala et al. 2006). Mientras que en los bosques nublados se observa una tendencia similar para Liquidambar styraciflua, en tanto para Quercus germana y Quercus xalepensis el crecimiento es menor. Existen varias explicaciones a estas diferencias, dentro de las cuales se encuentra el sitio, las características intrínsecas de la especie como la densidad específica de la madera, la competencia, el estadio de la sucesión en el cual se desarrollan las especies, entre otras.

Se tienen numerosas mediciones del incremento diamétrico instantáneo de varias especies tropicales, pero no reúnen los requisitos necesarios para desarrollar las curvas clásicas de incremento por tener datos de pocos árboles y clases diamétricas. Como en el bosque tropical Cerrado Boliviano (López et al. 2012), Sierra Norte de Puebla (CruzMárquez et al. 2011), especies tropicales como caoba, cedro, maculis, melia, teja y bojon (LópezTorres y Tamarit-Urías 2005), para especies del norte de Oaxaca (Manzano-Méndez et al. 2010) y de Campeche (López-Torres y Tamarit-Urías 2005). En estos trabajos se requiere del desarrollo de metodologías para el cálculo del crecimiento con pocas bases de datos. Una posibilidad es correlacionar el crecimiento de las diferentes especies que componen el bosque y medir los ritmos de una especie para todas las clases diamétricas, para conocer los ritmos de crecimiento de las otras especies que tienen poca información de crecimiento. Otra posibilidad es modelar los incrementos en función de los mismos en etapas tempranas hasta completar la curva. La derivación de las curvas de crecimiento con datos escasos de incremento debe de ser una prioridad ya que su utilidad práctica es necesaria en el desarrollo preliminar de planes de manejo forestal convencional y sustentable. El cálculo del crecimiento en volumen a través del diámetro debe replantearse para calcular mejor la productividad. Al respecto Navar (2010) en datos de incremento 
diamétrico de 15 árboles de selvas tropicales, encontró una relación negativa exponencial significativa del incremento diamétrico $\left(\frac{\partial D}{\partial t}\right)$, con la gravedad específica $(\rho)$, de la madera. Lo que indica que el incremento diamétrico es mayor en las especies de árboles tropicales de menor gravedad específica y menor en especies con altas gravedades específicas. Esto indica que los modelos de productividad que describen la ganancia en masa $\left(\mathrm{Mg} \mathrm{ha}^{-1}\right.$ año $\left.^{-1}\right)$, pueden presentar una menor variación que los clásicos modelos de incremento y crecimiento en volumen.

\section{CONCLUSIONES}

Los modelos de incremento y crecimiento al nivel del árbol individual, apoya el empleo de los modelos empíricos para proyectar el componente sigmoidal de especies de bosques tropicales. Se recomienda en futuras mediciones que el incremento diamétrico anual se realice en árboles individuales que cubran todas las clases diamétricas medidas en el inventario nacional forestal de la zona, para poder entender y modelar de mejor manera la aceleración inicial y final del crecimiento, para establecer los diámetros iniciales de referencia.

\section{LITERATURA CITADA}

Alder D, Synnott T (1992) Permanent sample plot techniques for mixed tropical forest. Oxford Forestry Institute, UK. Tropical Forestry Paper No. 25 124p.

Buongiorno J (2004) The use of Markov optimization models in the economic and ecological management of forest landscapes under risk. University of Wisconsin-Madison. Technical Series No 35. 25p.

Clutter JL, Forston JC, Pienaar LV, Brister GH, Bailey RL (1983) Timber management: A quantitative approach. Wiley, New York. 333p.

Corral-Rivas S, Návar-Cháidez J (2005) Análisis del crecimiento e incremento de cinco pináceas de los bosques de Durango, México. Madera y Bosques 11: 29-47.

Cruz-Márquez U, Negreros-Castillo P, López-Binquist C, Mize CW (2014) Crecimiento y producción de Trema micracantha (L) Blume, árbol para el papel amate. Revista Chapingo. Serie Ciencias Forestales y del Ambiente 20: 131-142.

FAO (2015) Food and Agriculture Organization. United Nations. Forest Statistics. Rome, Italy. 80p.

Haan CT (2003) Statistical methods in hydrology. lowa State University Press. Ames, lowa, USA. 378p.

Husch B, Kershaw JA, Beers TW (2003) Forest mensuration. 3rd Edition. John Wiley and Sons. New York, USA. 456p.

INF (2009) Inventario Nacional Forestal. Resultados del Inventario Nacional Forestal 2004-2006. Comisión Nacional Forestal. Guadalajara, México. 210p.

López-Ayala JL, Valdez-Hernández JI, Terrazas T, Vález-Lazalde R (2006) Crecimiento en diámetro de especies arbóreas en una selva mediana subcaducifolia en Colima, México. Agrociencia 40: 139-147.

López L, Villalba R, Peña-Claros M (2012) Ritmos de crecimiento diamétrico en los bosques secos tropicales: aportes al manejo sostenible de los bosques de la provincia biogeográfica del Cerrado Boliviano. Bosque (Valdivia) 33: 211-219.

López-Torres JL, Tamarit-Urías JC (2005) Crecimiento e incremento en diámetro de Lysiloma latisiliquum (L.) Benth. En bosques secundarios en Escárcega, Campeche, México. Revista Chapingo: Serie Ciencias Forestales y del Ambiente 11: 132-140. 
Lozano LA, Franco N, Bonilla JL (2012) Estimación del crecimiento diamétrico, de Anacardium excelsum (Kunth) Skeels, por medio de modelos no lineales, en bosques naturales del Dapartamento de Tolima. Bology Cientarum Museo Historia Natural 16: 19-32.

Manzano-Méndez F, Valdez-Hernández JI, López-López MA, Vaquera-Huerta H (2010) Crecimiento en diámetro de Zanthoxylum kellermanii P. Wilson en una selva perennifolia del norte de Oaxaca, México. Madera y Bosques 16: 25-33.

Merlin-Bermúdez E, Návar-Cháidez JJ (2005) Desarrollo de modelo de rendimiento e incremento para Quercus sideroxylla en bosques mixtos de Durango, México. Agrofaz 5: 875-882.

Návar J (2010) Measurement and assessment methods of forest aboveground biomass: A literature review and the challenges ahead. In: Momba M, Bux F (ed). Biomass. Sciyo: Rijeka, Croatia. pp: 27-64.

Návar J (2014) A stand-class growth and yield model for Mexico's northern temperate, mixed and multiaged forests. Forests 5: 3048-3069.

Návar J, Lizárraga-Mendiola L (2014) Tree ring growth and hydro-climatic variability in temperate dendrochronologies of northern Mexico. Agronomía Colombiana 32: 103-112.

Návar-Cháidez JJ, González-Elizondo MS (2009) Diversidad, estructura y productividad de bosques templados de Durango, México. Polibotánica 27: 69-85.

Návar-Cháidez JJ, Domínguez-Calleros PA (2013) Modelos de incremento y rendimiento sustentables para bosques de clima templado: ejemplos y aplicaciones para bosques Mexicanos. Revista Mexicana de Ciencias Forestales 18: 8-26.

Návar J, Domínguez-Calleros PA, Rodríguez-Flores FJ, de Hoogh R, Synnott T (2016) A stand growth and yield model for northern reforested stands of Mexico. Mathematical and Computational Forestry \& Natural Resource Sciences 8: 1-25.

Peng C (2000) Growth and yield models for uneven-aged stands: past, present and future. Forest Ecology and Management 132: 259-279.

Ramírez-Angulo H, Acevedo M, Atarof M, Torres-Lezama A (2009) Crecimiento diamétrico de especies arbóreas en un bosque estacional de los llanos Occidentales de Venezuela. Ecotropicos 22: 46-63.

Ricker M, del Río J (2004) Projecting diameter growth in tropical trees: a new modeling approach. Forest Science 50: 213-224.

Smith WR, Farrar Jr. RM, Murphy PA, Yeiser JL, Meldahl RS, Kush JL (1992) Crown and basal area relationships of open-growth southern pines for modeling competition and growth. Canadian Journal of Forest Research 22: 341-347.

Vanclay JK (1995) Growth models for tropical forest: A synthesis of models and methods. Forest Science 41: 7-42.

Williams-Linera G (1996) Crecimiento diamétrico de árboles caducifolios y perennifolios del bosques mesófilo de montaña en los alrededores de Xalapa. Madera y Bosques 2: 53-65.

Zeide B (1995) A relationship between size of trees and their number. Forest Ecology and Management 72 : 265-272.

Zeide B (1999) Pattern of height growth for southern pine species. Forest Ecology and Management 118: 183-196. 